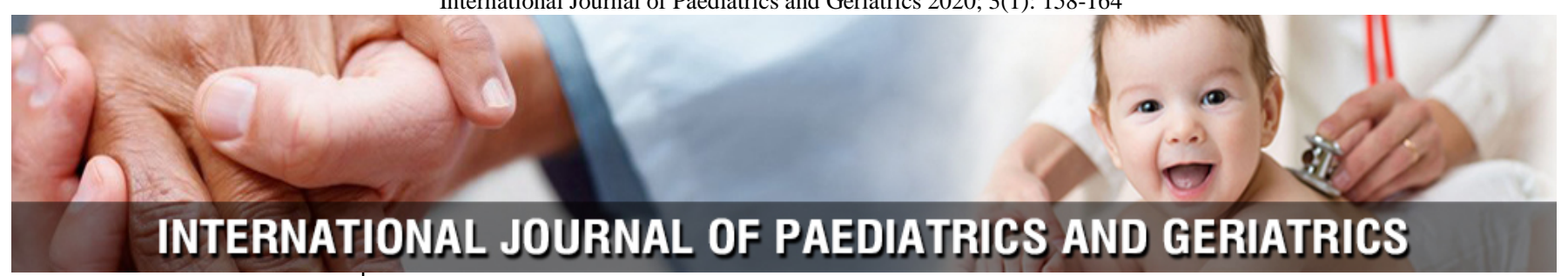

P-ISSN: 2664-3685

E-ISSN: $2664-3693$

www.paediatricjournal.com IJPG 2020; 3(1): 158-164

Received: 18-11-2019

Accepted: 19-12-2019

Rekha Bhattacharya PhD Scholar, Ph.D. Guide SRK University, Bhopal, Madhya Pradesh, India.

Dr. Bharati Batra PhD Scholar, Ph.D. Guide SRK University, Bhopal, Madhya Pradesh, India.

Corresponding Author: Rekha Bhattacharya PhD Scholar, Ph.D. Guide SRK University, Bhopal, Madhya Pradesh, India.

\section{"Effect of Helfer skin tap technique and expressed breast milk on response to pain among neonates during intramuscular injection"}

\author{
Rekha Bhattacharya and Dr. Bharati Batra \\ DOI: https://doi.org/10.33545/26643685.2020.v3.i1c.75
}

\begin{abstract}
Background: The aim of the study was to examine the effect of Helfer skin tap technique and expressed breast milk on response to pain among neonates during intramuscular injection of injection Vitamin K.

Design: Prospective randomized controlled trial.

Sample: 60 neonates for injection vitamin K after birth [20 in each group.

Control group (G1), experimental group Helfer technique (G2), EBM (G3)].

Material \& Methods: Sixty term neonates were enrolled for this study. The inclusion criteria were gestational age 37-40 weeks, birth weight 2.5-4 Kg, APGAR score more than 7 at 1 minute. The enrolled neonates were randomized into control group (G1) and two intervention groups- Heifer technique group (G2), EBM (G3). During intramuscular injection procedure was video graphed and pain score, Heart rate, respiratory rate and $\mathrm{SpO}_{2}$ are assessed by researcher. Pain score assessed using Neonatal Infant Pain Scale (NIPS).

Result: The mean pain score of the three groups G1, G2, G3 were 4.5, 3.55\& 3.19. Breast feeding had lowest mean pain score. Alterations in Heart rate, respiratory rate and $\mathrm{SpO}_{2}$ lowest in Heifer technique group.

Conclusion: Pain in new born should recognized and treated. EBM and Heifer technique before injection can be easily applied easily to alleviate pain response. Educational programmers for pain management in newborns should be conducted and guidelines can be prepared as institutional protocol.
\end{abstract}

Keywords: Helfer skin, intramuscular injection, pain, milk, expressed, institutional protocol

\section{Introduction}

Pain management in neonate is a very challenging issue in realm of neonatal care. 'Neonate do not feel pain' was a myth in the past. Neonates cannot verbalize their pain. They need to be observed by others for right assessment of pain and manage their pain. The nurses are the ones who assess the pain and alleviate it ${ }^{[1]}$.

Pain is a worldwide health problem and can cause complications which may be short term and long term. Short term complications may be increased heart rate, respiratory rate, blood pressure, decrease oxygen saturation, endocrine and biochemical changes such as hypoglycaemia, increased cortisol level, catecholamine release and a decrease in insulin are among reported problems in neonate ${ }^{[2]}$.

Pain in neonate due to procedures has been a concern in the last two decades. A neonate is subjected to on an average 7-17 procedure per day during the first 14 days of life after birth. During neonatal care neonates experience pain which includes intramuscular injections of Vitamin K, vaccination like Hep B, BCG, blood sampling, venepuncture etc ${ }^{[3]}$.

Pain pathways in cortical and subcortical centres necessary for pain perception are well developed which is proven and documented as opposed to popular myth of neonates do not feel pain. Neonates not only perceive pain but also exhibit behaviour responses to it by cry, grimaces, movements of arms and legs etc. Pain in neonates is mostly misunderstood, under diagnosed and under treated ${ }^{[4]}$.

Various pharmacologic and non-pharmacologic methods have been applied by the health care professionals for pain relief in neonates. Pharmacologic methods are judiciously used because of the potential side effects. So administering analgesics to neonates should be the last option for pain relief. Non Pharmacologic methods are valuable alternatives for effective 
pain relief. These include expressed breast milk/breast feeding, skin to skin contact, oral glucose/sucrose, massage, skin tapping, swaddling, non nutritive sucking and kangaroo mother care etc. From the first day of life, even healthy newborns are inevitably subjected to multiple painful interventions like Injection Vitamin $\mathrm{K}$ or Hepatitis $\mathrm{B}$ vaccines, heel lance etc ${ }^{[4-6]}$.

Breast milk contains higher concentration of tryptophan, a precursor of melatonin which is known to increase the level of beta-endorphin and could possibly be one of the mechanisms for the nociceptive effects of breast milk. Use of breast milk has additional benefit of promotion of breast feeding and it is cost effective too. Despite such advantages, breast milk is not regularly used for pain alleviation by health care centres ${ }^{[7]}$.

Marlow D.R \& Redding B.A (2013) coded that pain is a subjective experience and children responded to pain with psychological and behavioural reactions. Behavioural cues include increase irritability, restlessness, lack of appetite, pulling away, increase in heart rate and respiration often present with pain ${ }^{[8]}$.

In the article 'The Fifth vital sign: Implementation of Neonatal Infant Pain Scale by Gallo A M describes a Californian hospitals implementation of NIPS and the education provided to Nurses and its utilization in clinical practice. NIPS was found to be accurate in assessing pain and easy in application by nurses ${ }^{[9]}$.

Sweet tasting solutions are well described as effective strategies for pain relief in infants undergoing minor proceedings. Express breast milk (EBM) is considered as an alternative intervention to sweet solutions. It is readily available in clinical setting, naturally occurring and has many advantages. Vohra A conducted RCT to compare analgesic effect of expressed breast milk and 24\% sucrose solutions on procedural pain in 500 neonates. Either EBM or sucrose was given to neonates $2 \mathrm{mts}$ before Hep B Vaccine as intramuscular injection. PIPP was used to assess pain score of neonates after 30 sec respectively. The mean PIPP score at $30 \mathrm{sec}$ were 5.7 in EBM group and 6.9 in sucrose s group $(\mathrm{p}<0.001)^{[10]}$.

A true experimental research study conducted by Sivapriya et al. to assess the effectiveness of Helfer technique on pain associated with intramuscular injection among neonates. The study result showed that there is significant difference in the pain score during intramuscular injection with Helfer technique ${ }^{[11]}$.

Nurses working in the labour room, maternity ward or neonatal care unit are the best person to identify and observe signs and symptoms of pain and take measures to reduce or alleviate pain. The present study assesses the efficacy of expressed breast milk and Helfer technique on neonatal pain during Intramuscular injection.

\section{Problem Statement}

"Effect of expressed Helfer skin tap technique and breast milk on response to pain among neonates during intramuscular injection”.

\section{Objectives}

1) To assess the pain level of neonates during intramuscular injection in the experimental groups and control group.

2) To compare the pain level of neonates in the experimental groups and control group.

\section{Operational definition}

Pain: An unpleasant sensory and emotional experience associated with actual or potential tissue damage during intramuscular injection.

Efficacy: Make a desired or intended result in reducing pain sensation in neonate during intramuscular injections of Vitamin K

Expressed Breast Milk: 2ml of milk expressed from mothers breast manually.

Helfer Skin Tap Technique- Helfer Skin Tap Technique: - It is a technique in which the investigator is tapping over the intramuscular injection site with the palmer aspect of fingers 16 times before insertion and 3 counts during the procedure within $20 \mathrm{sec}$.

Neonates- New born up to the age of 28 days

\section{Hypothesis}

Ho: There is no difference in mean pain score during intramuscular injection among neonates who are given Helfer skin tapping before injection and those who are given EBM.

\section{Methodology}

Research Approach: Quantitative Research Approach

\section{Research design}

True experimental post test only design. Composed of three randomly assigned groups. Three group consists of

G1: Control group Neonates are given injection Vitamin K with standard technique.

G2: Helfer ST Neonates are given tactile stimulation with Helfer skin tapping before intramuscular injection of Vitamin K

G3: EBM Neonates are given $2 \mathrm{ml}$ of expressed breast milk before intramuscular injection of Vitamin K

Research setting: Labour room of a tertiary hospital Pune, Maharashtra.

\section{Sampling Technique}

Purposive sampling to select study subjects who fulfils the inclusion criteria. Simple random sampling technique (Lottery method) will be used to allocate the neonates into control group G1 and experimental groups G2, G3.

Sample Size: 60. (20 in G1 control group \& 20 each in experimental groups G2, G3).

\section{Inclusion criteria}

a. Neonates born by vaginal delivery or LSCS will be taken for the study

b. Neonates available for sampling during data collection period.

\section{Exclusion criteria}

A. Neonates who are born $<2.5 \mathrm{~kg}$ wt, has any respiratory distress or any severe problems.

\section{Variables under study}

Independent variable: In this study tactile stimulation with Helfer skin tap technique and expressed breast milk feeding before administration of injection vitamin $\mathrm{K}$ intramuscularly.

Dependent variable: The dependent variables in this study are pain score during vitamin $\mathrm{K}$ injection. 


\section{Tool Used for data collection}

The Neonatal Infant Pain Scale (NIPS) is used for assessing the pain after vaccination.

\section{Maximum Score: $-7 \quad$ Minimum Score: -0}

No/Mild Pain 0-2, Moderate Pain 3 -4, Severe Pain $>4$.

\section{Ethical consideration}

Permission has been obtained from the ethical committee of the institution and the written consent has been obtained from the participants.

Procedure for data collection

- Formal permission has been obtained from the administration

- Infants have been identified as per inclusion criteria

- Collected baseline data

- The intervention was done as per the groups. The procedure was video recorded. Pain assessment done by using NIP scale by the researcher for all the groups.

\section{Result \& analysis of data}

Section I: Demographic variables of neonates participating in the study

Table 1: Distribution of the neonates as per demographic variables of Control group G1, Experimental groups (G2, G3)

\begin{tabular}{|c|c|c|c|c|c|c|c|c|}
\hline \multirow[t]{2}{*}{ S. No. } & \multirow[t]{2}{*}{ Variable } & \multirow[t]{2}{*}{ Category } & \multicolumn{2}{|c|}{ G1 Control Group N=20 } & \multicolumn{2}{|c|}{ G2 Helfer S T N=20 } & \multicolumn{2}{|c|}{ G3 EBM N=20 } \\
\hline & & & (F) & $(\%)$ & (F) & $(\%)$ & (F) & $(\%)$ \\
\hline \multirow{4}{*}{1.} & \multirow{4}{*}{$\begin{array}{l}\text { Gestation Age } \\
\text { in weeks }\end{array}$} & 37 Weeks & 5 & 25 & 6 & 30 & 8 & 40 \\
\hline & & 38 Weeks & 9 & 45 & 9 & 45 & 5 & 25 \\
\hline & & 39 Weeks & 6 & 30 & 4 & 20 & 6 & 30 \\
\hline & & 40 Weeks & 0 & 0 & 1 & 5 & 1 & 5 \\
\hline \multirow{4}{*}{2.} & \multirow{4}{*}{ Weight } & & 2 & 10 & 1 & 5 & 1 & 5 \\
\hline & & 2.0- $2.5 \mathrm{Kg} 2.6-3.0 \mathrm{Kg} 3.1-3.5$ & 8 & 40 & 11 & 55 & 7 & 35 \\
\hline & & $\mathrm{Kg}, 3.6-4.0 \mathrm{Kg}$ & 10 & 50 & 8 & 40 & 10 & 50 \\
\hline & & & - & - & - & - & 2 & 10 \\
\hline \multirow{2}{*}{3.} & \multirow{2}{*}{ Gender } & Male & 9 & 45 & 13 & 65 & 11 & 55 \\
\hline & & Female & 11 & 55 & 7 & 35 & 9 & 45 \\
\hline \multirow{2}{*}{4.} & Mode of & Normal & 12 & 60 & 14 & 70 & 11 & 55 \\
\hline & Delivery & LSCS & 8 & 40 & 6 & 30 & 9 & 45 \\
\hline \multirow{2}{*}{5.} & Anesthesia/ & Yes & 1 & 5 & 7 & 35 & 8 & 40 \\
\hline & Analgesia & No & 19 & 95 & 13 & 65 & 12 & 60 \\
\hline \multirow{2}{*}{6.} & \multirow{2}{*}{ Complications } & Yes & 0 & - & 2 & 10 & - & - \\
\hline & & No & 20 & 100 & 18 & 90 & 20 & 100 \\
\hline
\end{tabular}

Figures describes sample characteristics

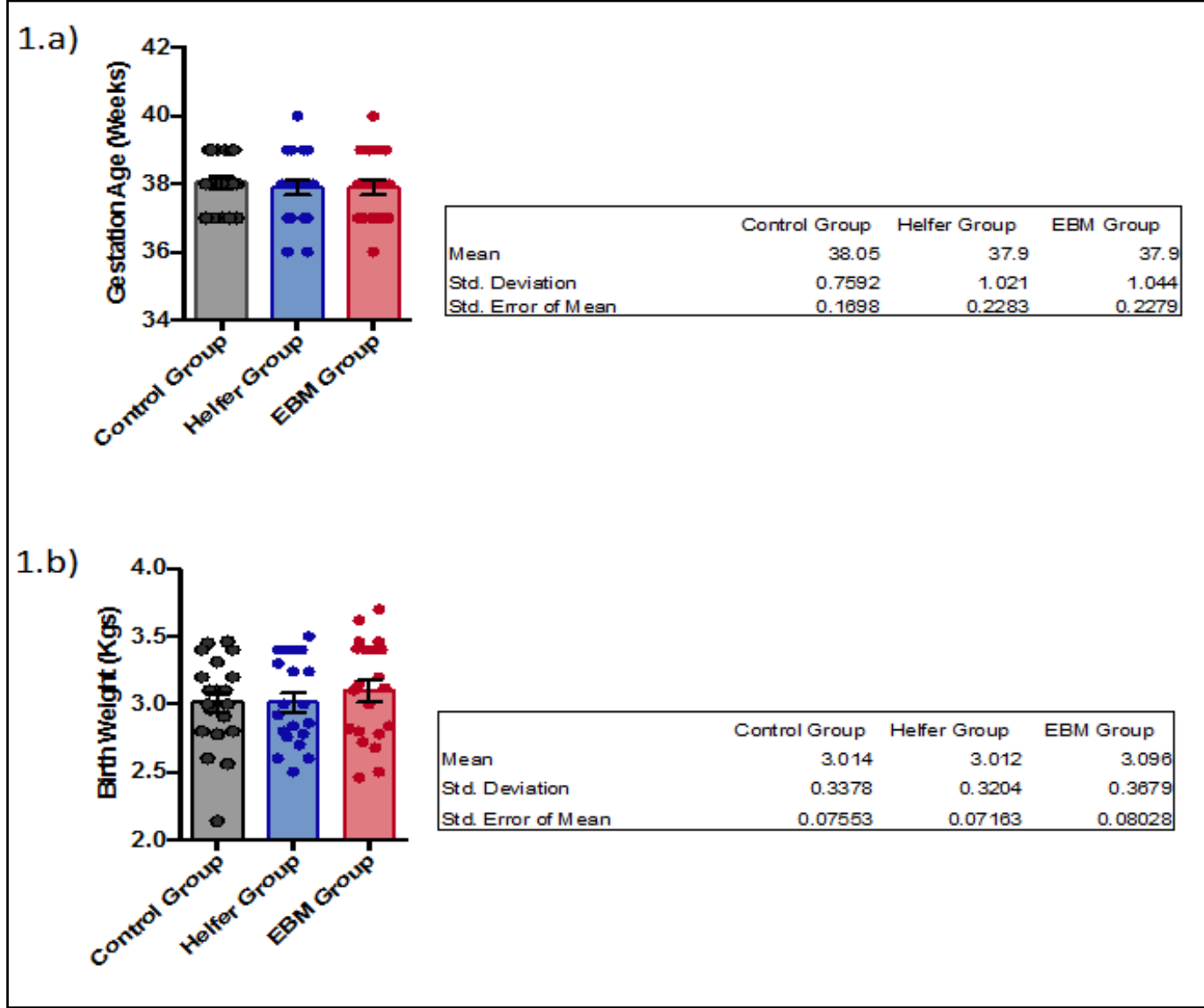

Fig 1: Sample characteristics in terms of Gestation age and Birth weight 


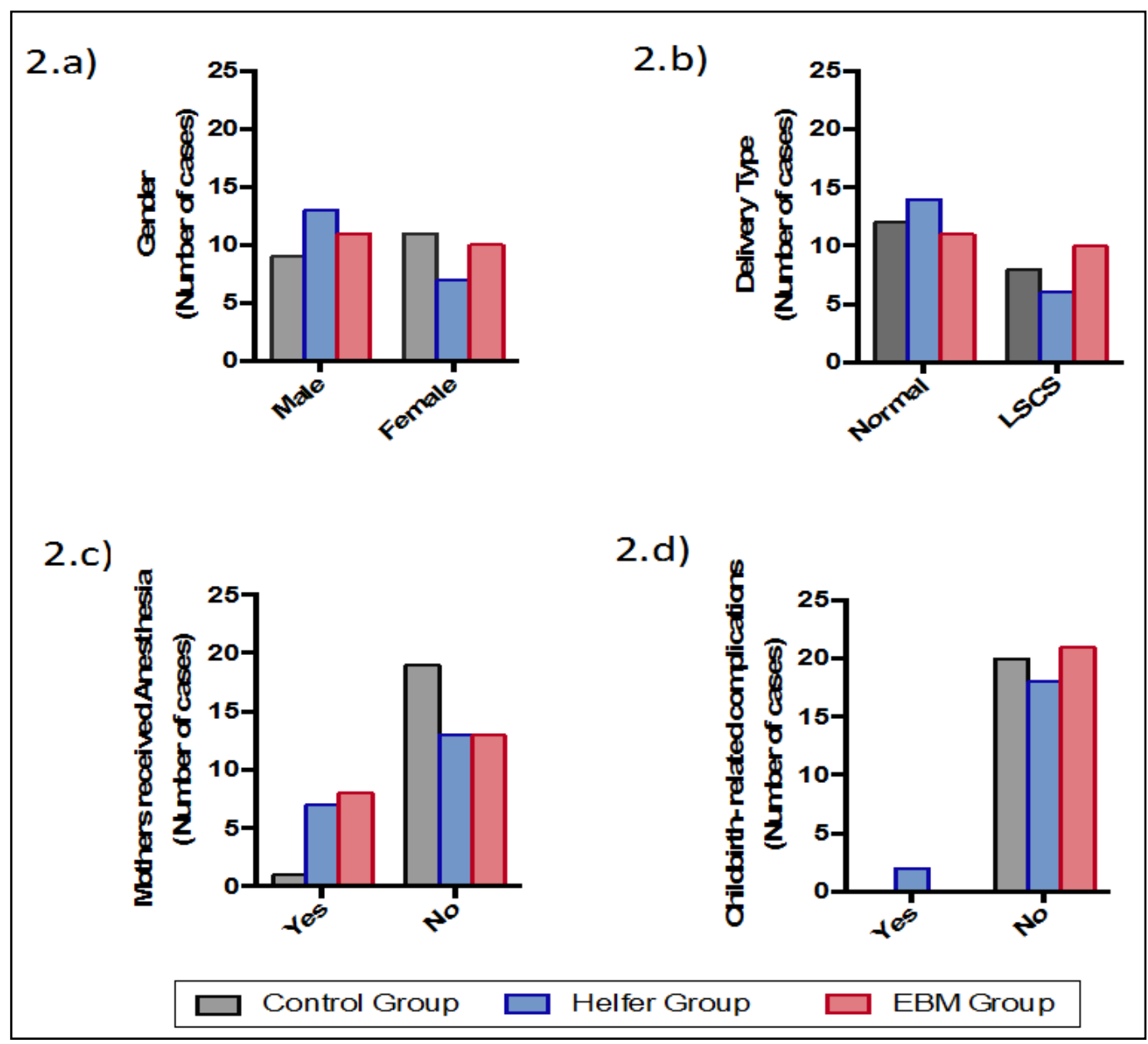

Fig 2: Sample characteristics in terms of Gender, type of delivery, Analgesia used \& complication

Section II: Assessment of Heart rates, respiration and SpO2 among control and experimental groups

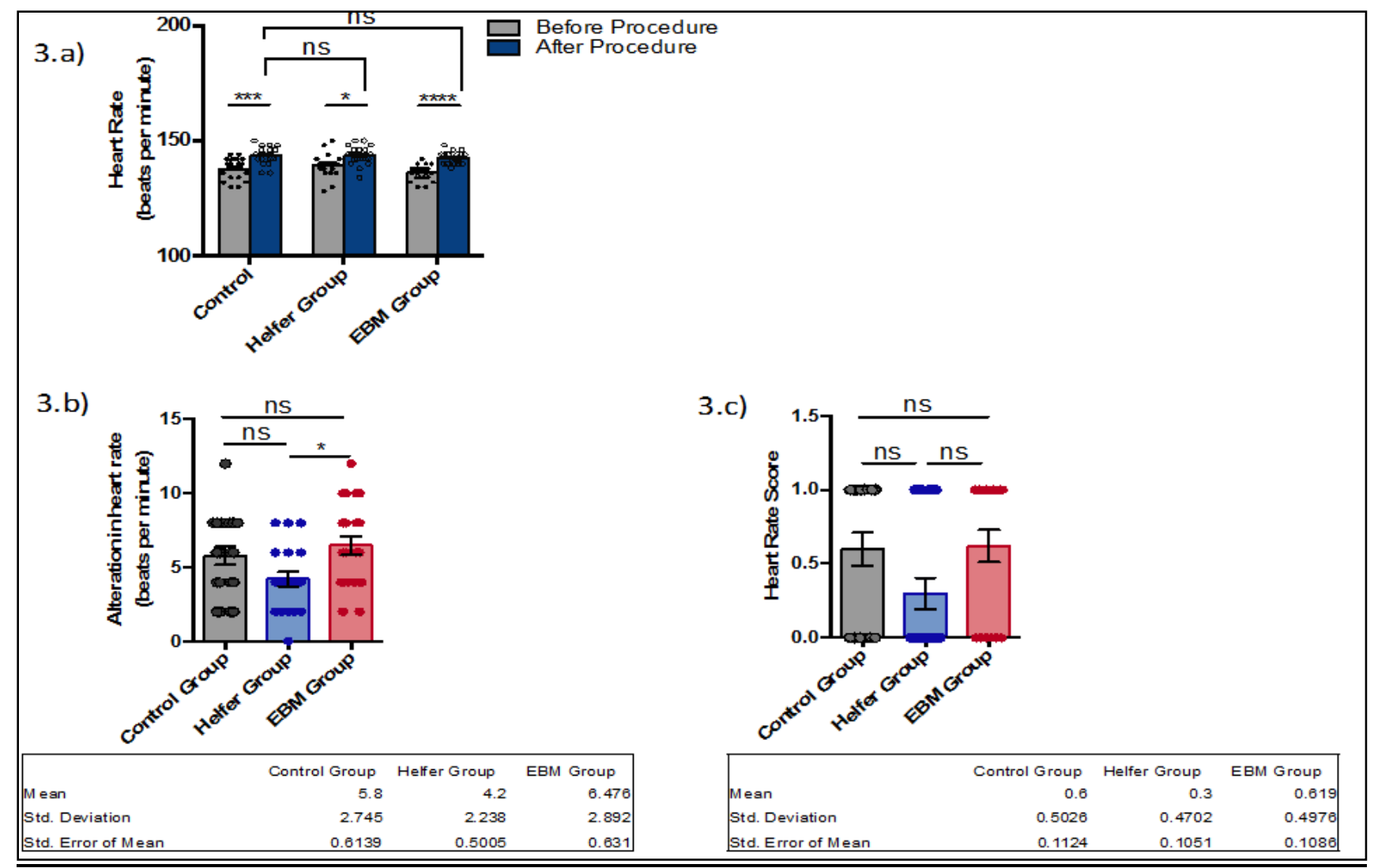

Fig 3: Heart rate \& its alteration 


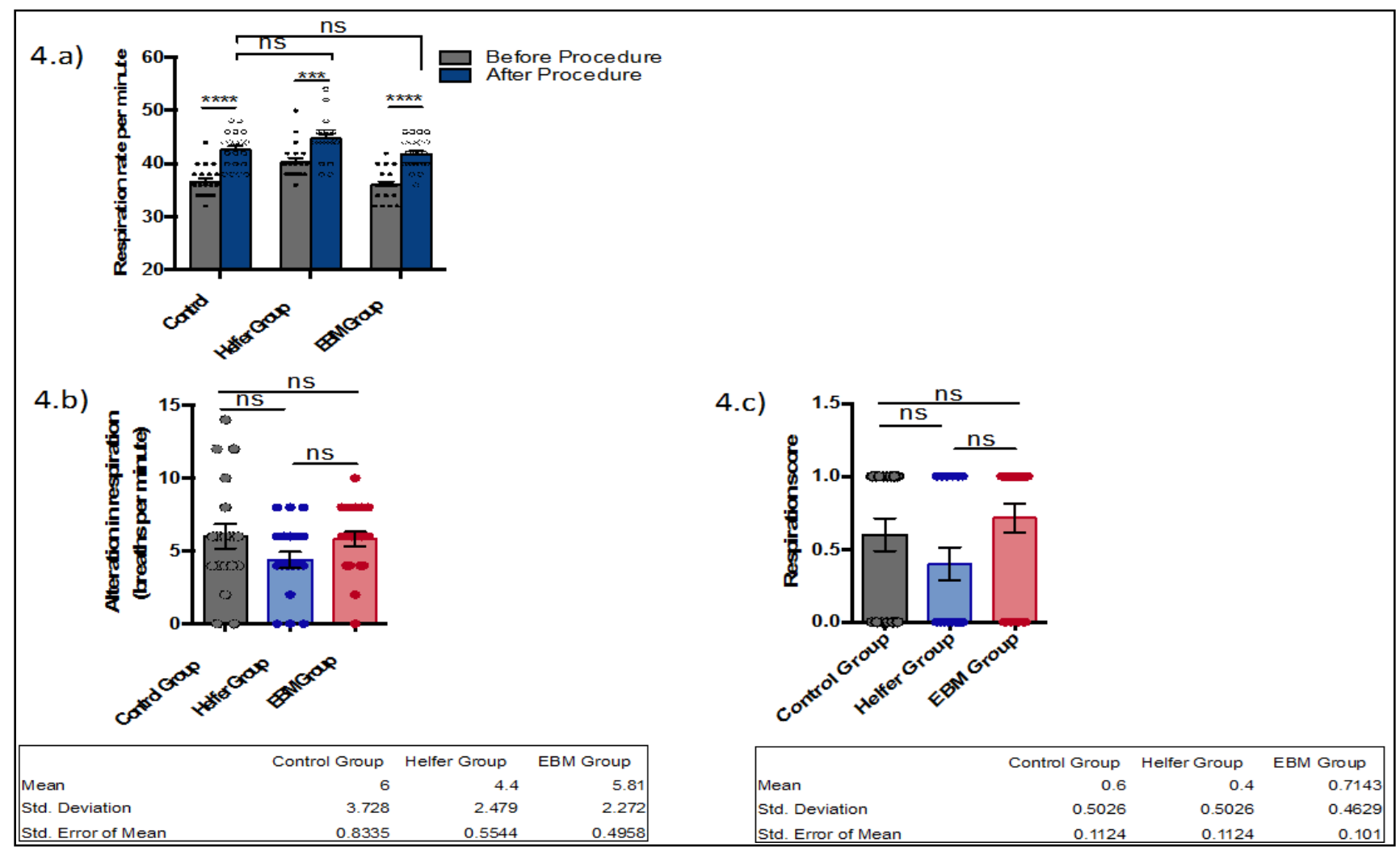

Fig IV: Respiration rate \& its alteration

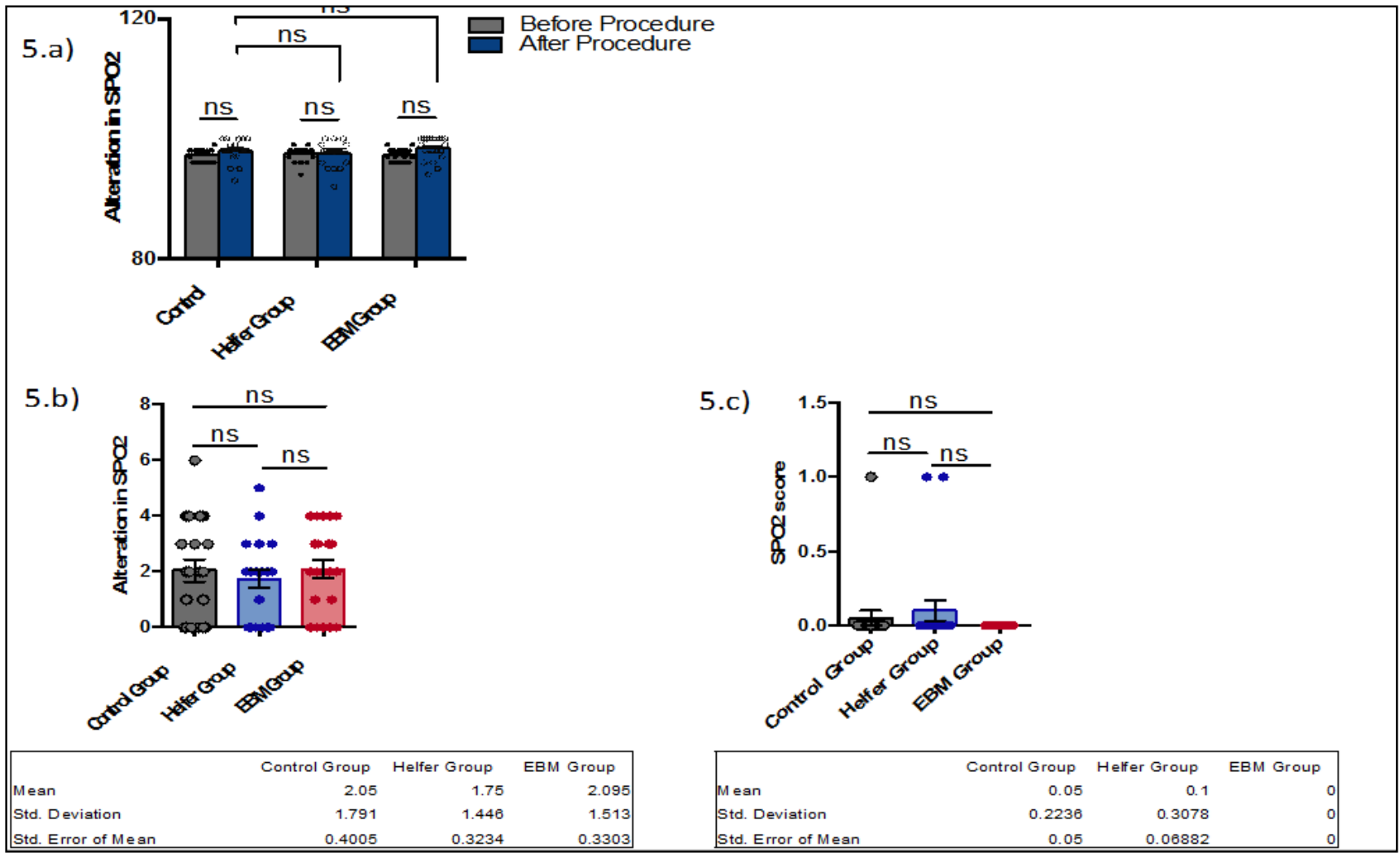

Fig V: $\mathrm{SpO}_{2}$ \& its alteration

Section III: Pain Score In Control Group (G1) and Experimental Groups (Heifer techniqueG2, EBM G3) 


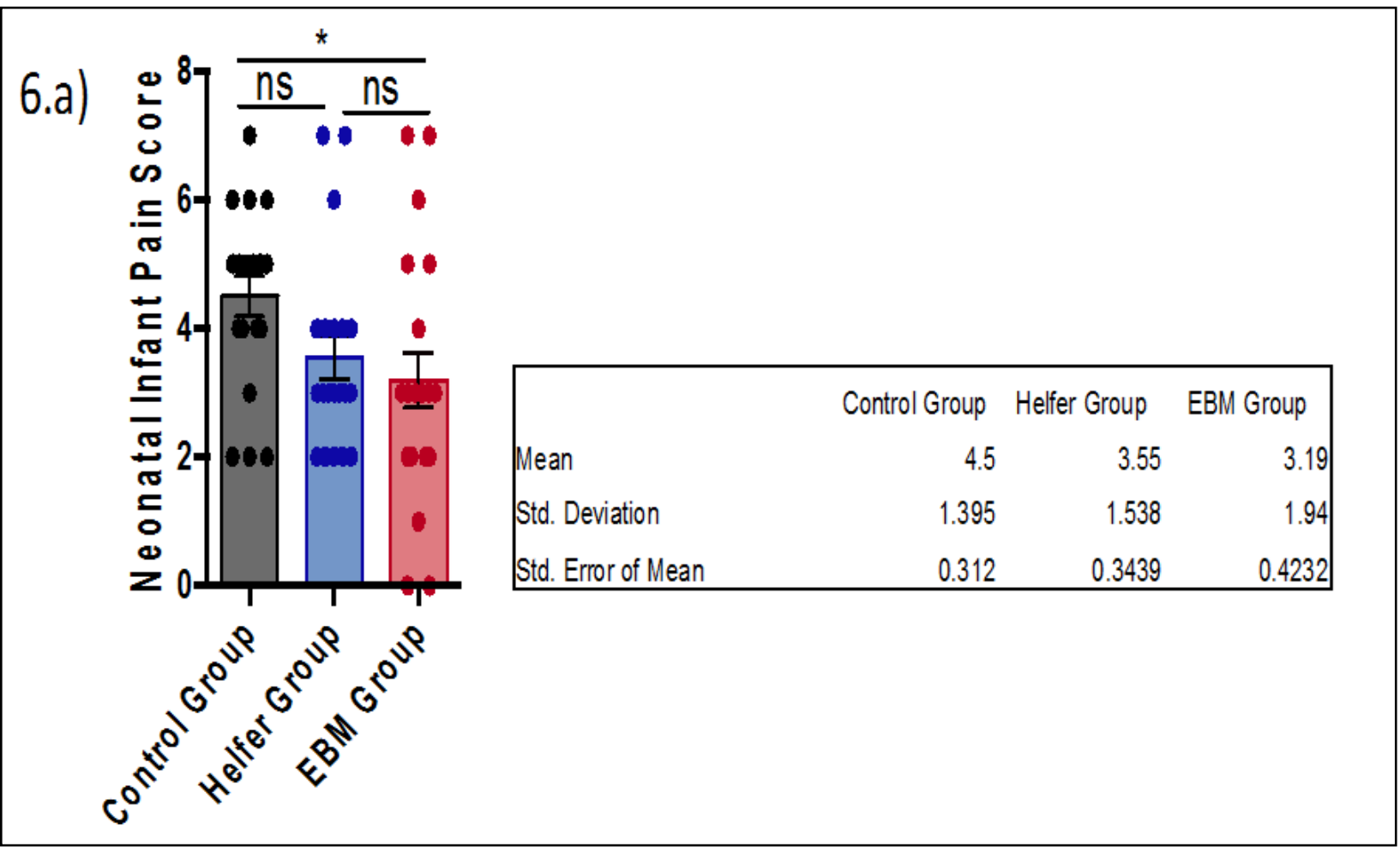

Fig VI: Comparison of mean pain score \& SD in control group (G1) and experimental groups (Heifer technique G2, EBM G3)

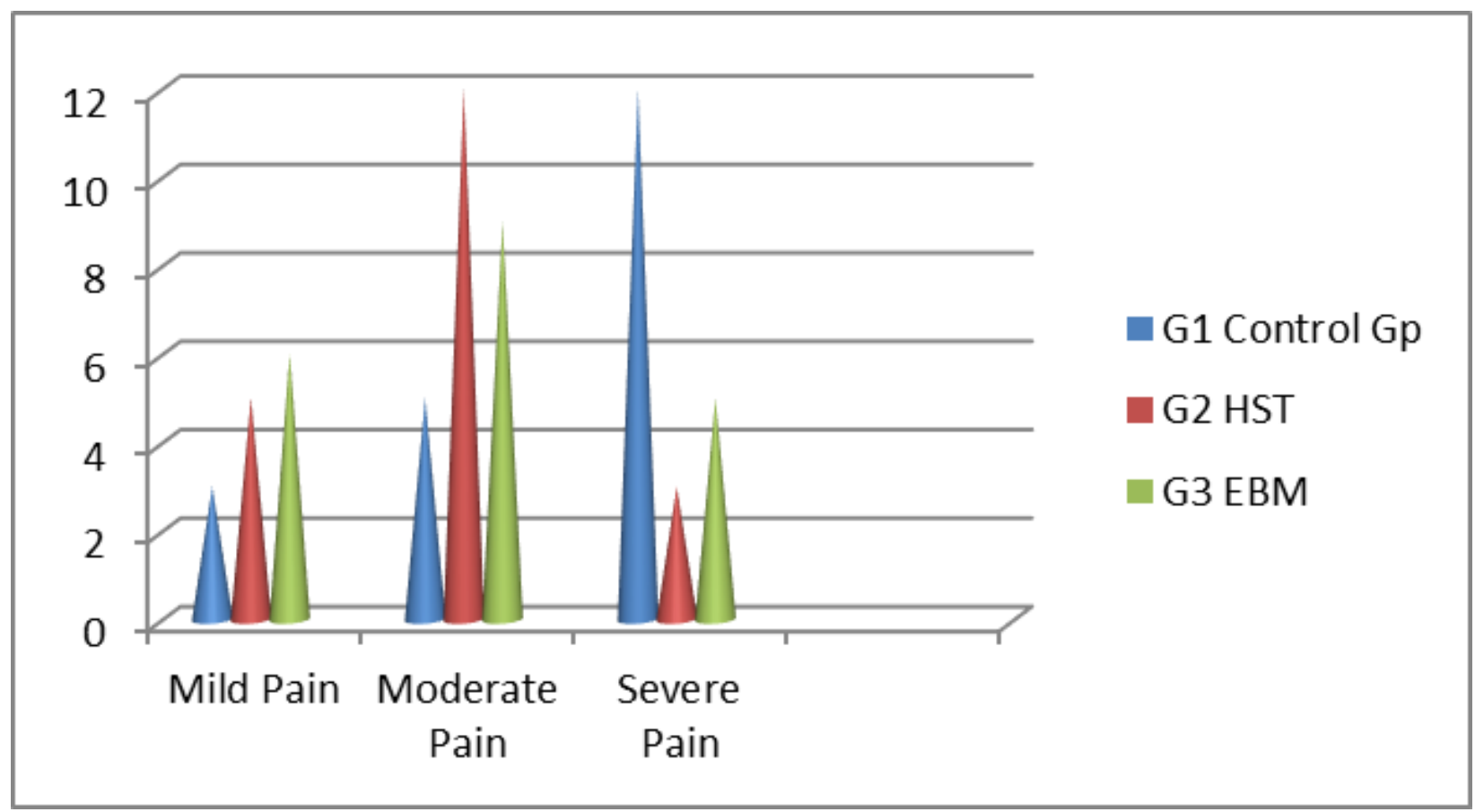

Fig VII: Pain Score during intramuscular injection in control group (G1) and experimental groups (Heifer Techniqueg2, EBM G3)

\section{Findings of the study}

Sixty full term neonates were taken for the study (20 in control and 20 each in experimental groups). No differences were observed between the groups with regard to baseline variables. Chi square test is done to investigate the homogeneity of the group.

The mean and SD of pain scores during intramuscular injection were 4.5 \& 1.39 in Control group (G1), 3.55 \& 1.538 and 3.19 \& 1.94 in Heifer and EBM group respectively. The lowest mean pain score recorded in the EBM group (G3).

When the groups were compared for all the physiological parameters, mean alteration in the heart rate, respiratory rate and $\mathrm{SpO}_{2}$ are lowest in Heifer group (G2).

\section{Recommendations}

1. Study findings can be integrated into nursing practice.

2. Studies on other non-pharmacologic methods can be conducted with large sample size.

3. Comparison can be done with other painful procedures like intravenous procedure, heel lance.

4. Nurses need to have knowledge on assessment, treatment and prevention of complication related to pain. 


\section{Conclusion}

The findings of the study showed that simple measures like expressed breast milk and tactile stimulation by Helfer technique may be done before painful procedure to control procedural pain in neonate. More studies are needed to investigate other painful procedures and non-pharmacologic methods for neonates. Considering the fact that Expressed breast milk and Heifer skin tap technique are natural, useful and cost free intervention and do not need any specific facility, these methods are suggested for pain control during painful procedures in neonates.

\section{References}

1. Merchant A. Neonate do not feel pain; a critical review of the evidence Bioscience horizons, 2014.

2. Boroujeni Zhargam A, Elsagh A, Mohammadizadeh M. The effects of Massage and Breastfeeding N Response to venipuncture pain among hospitalized neonates, Iranian Journal of nursing and midwifery research. 2017; 22:308-12.

3. Cruz M.D, Fernandes A.M, Oliveira C.R. Epidemiology of painful procedures performed in neonate a systematic review of observational studies, European pain federation-EFIC, 2015.

4. Mathew P.J, Mathew J.L. Assessment and management of Pain in Infants Postgrad Med J. 2003; 79:438-443.

5. Srouji R, Ratnapalan S, Suzan Schneeweiss. Pain in children: assessment and No pharmacological Management. 2010; 1.

6. Mathai S, Natarajan N, Rajalakshmi N.R. A comparative study of non-pharmacological methods to reduce pain in neonates Indian Pediatrics. 2006; 43:1070-74.

7. S Sujatha, Samson R, Sundaresan. Breast milk for neonatal vaccination pain, The nursing Journal of India. 2018; CIX(6):249-252.

8. Marlow D.R, Redding B.A. Text book of paediatric nursing $6^{\text {th }}$ ed Saunders publication, 2013.

9. Gallo A.M. The fifth vital sign: Implementation of neonatal infant pain scale JOGNN, 2003; 32:199-206.

10. Vohra A, Purani C, Mehariya M K, Shah B, Neonatal Analgesia: Effect of sucrose solution versus breastfeeding in procedural pain, Paediatric on call (Serial online), (cited 2017 Oct-Dec1), 2017.

11. S Sivapriya, Leena Kumari. "Effectiveness of Heifer skin tap technique on pain during intramuscular injection among neonates", International Journal of science \& research, www.ijsr.net. 2015; 4:4.

12. McGrath. Pain in children. New York: Guit fort publications. 1990, 3.

13. Ball J.W, Binder C, Text Book of Pediatric Nursing $4^{\text {th }}$ Ed New Delhi, Pearson Education, 2001.

14. Dhulkhed Vithal K, Dhorigol M G, Rajesh Mane et al. Basic statistical concepts for sample size estimation: Indian Journal of Anesthesia. 2008; 52(6):788-793. 